\title{
Factors associated with the refusal of surgery and the associated impact on survival in patients with rectal cancer using the National Cancer Database
}

\author{
Alex R. Coffman ${ }^{1}$, Randa Tao ${ }^{2}$, Jessica N. Cohan ${ }^{3}$, Lyen C. Huang ${ }^{3}$, T. Bartley Pickron ${ }^{3}$, \\ Anna M. Torgeson ${ }^{2}$, Shane Lloyd ${ }^{2}$ \\ ${ }^{1}$ Department of Radiation Oncology, Loma Linda University Medical Center, Loma Linda, CA, USA; ${ }^{2}$ Department of Radiation Oncology, \\ Huntsman Cancer Institute, Salt Lake City, Utah, USA; ${ }^{3}$ Department of Surgery, Huntsman Cancer Institute, Salt Lake City, Utah, USA \\ Contributions: (I) Conception and design: AR Coffman, S Lloyd; (II) Administrative support: AR Coffman; (III) Provision of study materials or \\ patients: AR Coffman, S Lloyd; (IV) Collection and assembly of data: AR Coffman; (V) Data analysis and interpretation: AR Coffman, S Lloyd; (VI) \\ Manuscript writing: All authors; (VII) Final approval of manuscript: All authors. \\ Correspondence to: Alex R. Coffman, MD. Department of Radiation Oncology, Loma Linda University Medical Center, 11234 Anderson Street, Suite \\ B121, Loma Linda, CA 92354, USA. Email: AlexCoffmanMD@gmail.com.
}

Background: Surgical resection is an integral component of the curative-intent treatment for most patients with non-metastatic rectal cancer. However, some patients refuse surgery for a number of reasons. Utilizing the National Cancer Database (NCDB), we investigated the sociodemographic and clinical factors associated with patients who were coded as having been offered but refused surgery, and the factors affecting overall survival (OS) in these patients.

Methods: Adult patients with adenocarcinoma of the rectum (excluding T1N0M0 and M1 disease) diagnosed from 2004 to 2015 were analyzed in this retrospective cohort study. Logistic regression was performed to identify factors associated with refusal of surgery. OS of patients refusing surgery was compared using Kaplan-Meier estimate, log-rank test, propensity score matching, and proportional hazards regression.

Results: A total of 55,704 patients were identified: 54,266 received definitive surgery (97.4\%) and 1,438 refused (2.6\%). Of patients refusing surgery, 135 (9.4\%) were stage I, 709 (49.3\%) were stage II, and 594 $(41.3 \%)$ were stage III. Patients were more likely to refuse surgery as the study period progressed $(\mathrm{P}<0.01)$. Factors associated with refusal of surgery on multivariate analysis include: age $\geq 70$ years, Black race, nonprivate insurance, and tumor size greater than $2 \mathrm{~cm}$ (all values $\mathrm{P} \leq 0.01$ ). The 5 -year OS was $61.6 \%$ for the surgery cohort and $35.7 \%$ for the refusal cohort. In the propensity matched groups, median survival was 84.2 months in patients who received definitive surgery compared to 43.7 months in patients who refused surgery. As an index for comparison, patients who refused surgery but received both radiotherapy and chemotherapy had a median survival of 48.5 months. Among patients that refused surgery, those that received radiotherapy alone, chemotherapy alone, or radiotherapy and chemotherapy (compared to no treatment) experienced a survival benefit (all values $\mathrm{P} \leq 0.01$ ).

Conclusions: In patients with non-metastatic adenocarcinoma of the rectum reported in the NCDB, age, race, and insurance status were associated with refusal of surgery. Refusal of surgery was more common in the later years of the study. Survival is poor in patients who refused surgical resection.

Keywords: Rectal cancer; refusal; National Cancer Database (NCDB)

Submitted Oct 11, 2020. Accepted for publication Jun 08, 2021.

doi: 10.21037/jgo-20-437

View this article at: https://dx.doi.org/10.21037/jgo-20-437

(C) Journal of Gastrointestinal Oncology. All rights reserved. 


\section{Introduction}

Approximately 45,230 people in the United States will be diagnosed with rectal cancer in 2021 (1). Surgical resection is an integral component of curative-intent treatment for patients that present with non-metastatic disease. The "watch and wait" approach is a promising organpreservation strategy for select patients who achieve a complete clinical response after chemoradiotherapy (2-6), but the possible increased risk of local and/or distant recurrence compared to standard surgical resection is unknown (7). Therefore, surgery with total mesorectal excision (TME) is the foundation of curative treatment for a majority of patients with localized rectal cancer, excluding well-selected patients with clinical T1 disease that can be treated with local excision alone (8-10). Some patients do not receive surgery as part of their rectal cancer treatment either because they are not surgical candidates or because they refuse surgery.

The decision to decline cancer treatment is an interesting and understudied phenomenon. Specifically, the refusal of surgery has been associated with poor survival outcomes in a number of disease sites (11-14). There are many documented factors that affect the decision for a patient to decline cancer treatment in general including fear of side effects, emotional impact of diagnosis, poor communication, and the desire for control over the decision-making process (15). There are many other potential reasons as to why a patient may elect to not undergo surgery including the need to care for another family member, loss of income that would devastate the family, and no resources for postoperative care. In terms of the morbidity associated with surgery in rectal cancer, one study compared a "watch and wait" group versus a TME group. The "watch and wait" group showed better physical and cognitive function, better physical and emotional roles, better global health status, and fewer problems with defecation and sexual and urinary tract function compared to the TME group (16). It is understandable that some patients may be hesitant to undergo surgery, especially when temporary or permanent stomas are required as in patients with low-lying tumors or sphincter involvement.

The purpose of this study was to investigate factors associated with patients who decline rectal cancer surgery and the subsequent impact of this decision on overall survival (OS). This clinical information could help practitioners counsel and better understand the possible reasons why a patient may decline a recommended surgical intervention. We conducted an analysis using the National Cancer Database (NCDB) to investigate the sociodemographic and clinical factors associated with patients who were coded as having refused surgery and compared the survival of these patients to those that received definitive surgery for the treatment of their nonmetastatic rectal cancer using propensity score matching. We present the following article in accordance with the Strengthening the Reporting of Observational studies in Epidemiology (STROBE) reporting checklist (available at https://dx.doi.org/10.21037/jgo-20-437).

\section{Methods}

\section{Data source}

This study was exempt from oversight by the Institutional Review Board as there is no identifiable patient information in the NCDB. The NCDB is a joint project of the Commission on Cancer $(\mathrm{CoC})$ of the American College of Surgeons and the American Cancer Society. This nationwide, facility-based, comprehensive clinical surveillance resource oncology data set currently captures $70 \%$ of all newly diagnosed malignancies from more than 1,500 CoC-accredited facilities in the United States annually. The accreditation requires an annual $90 \%$ followup rate for all eligible patients diagnosed within five years. The American College of Surgeons and the CoC have not verified and are not responsible for the analytic or statistical methodology employed, or the conclusions drawn from these data by the investigator (17).

\section{Cohort selection}

There are 264,184 patients with rectal cancer (International Classification of Diseases for Oncology, $3^{\text {rd }}$ Edition, topography code C209) documented in the NCDB between 2004 and 2015. We excluded patients if they were diagnosed with clinical stage 0 or clinical stage IV disease, or if the clinical stage was unknown $(n=131,238)$. Patients with clinical T1N0 disease were also excluded as these patients can potentially be treated with local excision alone $(n=26,484)$. Patients were also excluded if the histology was anything other than adenocarcinoma $(n=12,664)$. In terms of surgery, patients were excluded if they did not receive definitive surgery or if this information was unknown $(n=16,962)$. Local excision was not considered a definitive surgery. Patients were also excluded if the reason they did 


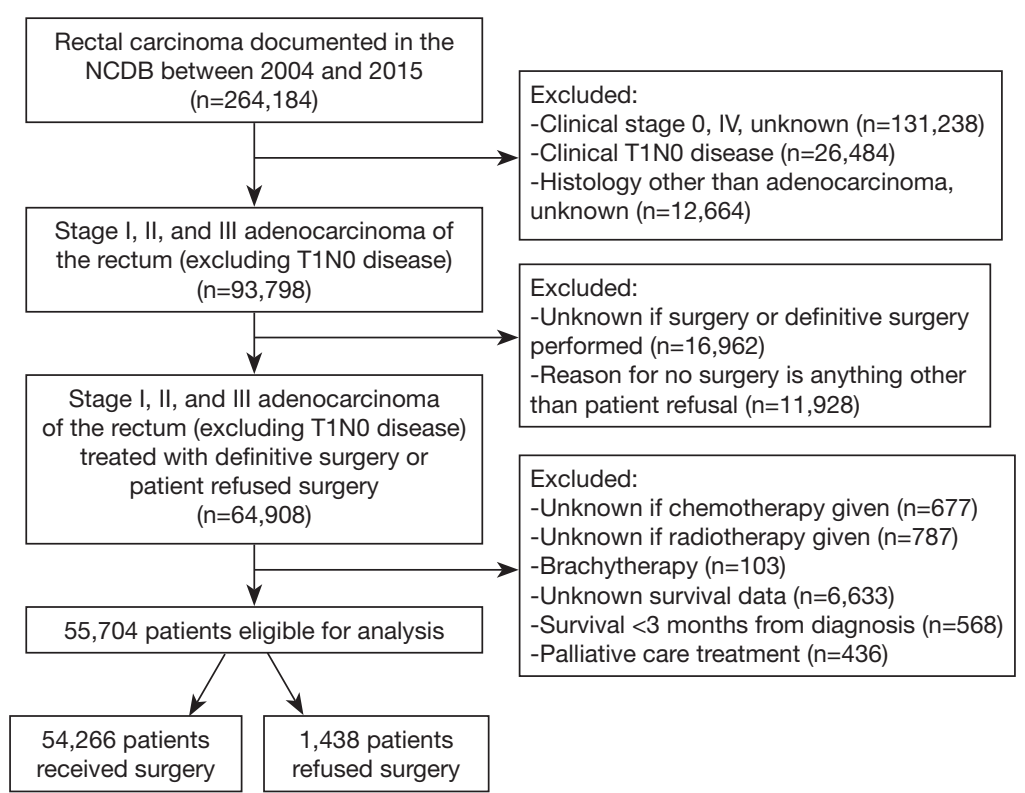

Figure 1 Patient selection diagram. NCDB, National Cancer Database.

not receive surgery was anything other than patient refusal $(\mathrm{n}=11,928)$. Of the patients that did not receive surgery for reasons other than refusal, $81.3 \%$ were coded as surgery not being part of the planned first course of treatment and $12.4 \%$ were due to patient risk factors (comorbidities, advanced age, tumor progression, etc.). A patient was coded as having refused surgery, according to the NCDB registry coding instructions for the reason no surgery was performed, if "the patient refused recommended surgical treatment, made a blanket refusal of all recommended treatment, or refused all treatment before any was recommended." Refusal of chemotherapy and radiotherapy are coded distinctly from not being offered or simply not receiving these treatments, as for surgery. Patients were excluded if it was unknown whether chemotherapy $(n=677)$ or radiotherapy $(n=787)$ was given, and if the patient received brachytherapy $(n=103)$. Finally, patients were excluded if survival data was unknown $(\mathrm{n}=6,633)$, if survival was less than 3 months from diagnosis $(\mathrm{n}=568)$, or if the patient was given palliative treatment $(n=436)$. A patient selection diagram of the patient inclusion criteria is shown in Figure 1. This left a population of 55,704 patients eligible for analysis. The final cohort of patients had non-metastatic adenocarcinoma of the rectum (excluding clinical T1N0 disease) treated with definitive surgery or the patient declined surgery with known chemotherapy and radiotherapy treatment and complete follow-up data.

\section{Covariates}

Demographic covariates included patient age (18-49, 50-69, $\geq 70$ years), sex (male, female), race (White, Black, Hispanic, other), insurance status (private, no insurance, Medicaid, Medicare), Charlson Comorbidity Index (CCI) $(0,1,2+)$, geographic region (Northeast, South, Midwest, West), income (<\$30,000, \$30,000-34,999, \$35,000-45,999, $\geq \$ 46,000)$, percentage of residents without a high school degree ( $\geq 29 \%, 20-28.9 \%, 14-19 \%,<14 \%$ ), type of treatment facility (community, academic/research), distance from treatment facility $(<50,50-200,>200$ miles $)$, urban density (metro/urban, rural), and year of diagnosis (20042015). Clinical covariates included clinical $T$ category (T1, T2, T3, T4), clinical $\mathrm{N}$ category (N1, N2, N3), tumor size $(\leq 2.0,2.1-5.0,5.1-10.0,>10.0 \mathrm{~cm})$, radiotherapy dose $(\leq 5,040,5041-5,400,>5,400 \mathrm{cGy})$, and treatment with chemotherapy and/or radiotherapy. After excluding patients with unknown stage, unknown histology, and unknown treatments, only $3.5 \%$ of values were missing across all variables. Therefore, no imputation was performed.

\section{Statistical analysis}

Characteristics between those patients who received definitive surgery and those that elected to forego surgery were analyzed. Chi-square analysis was used to compare 
the above demographic and clinical variables between these two cohorts. Univariate and multivariate logistic regression modeling was used to generate odds ratios (OR) with corresponding $95 \%$ confidence interval (CI) to identify factors predictive of receiving definitive surgery or declining surgery. Variables were included in the multivariate analysis if the $\mathrm{P}$ value on univariate analysis was $<0.20$, or if the inclusion of the variable resulted in a $10 \%$ or greater change in the outcome $(18,19)$. The year of diagnosis was analyzed using univariate and multivariate logistic regression as a continuous variable to determine if refusal of surgery changed over the 2004-2015 study period. There were an insufficient number of observations to run a CochraneArmitage test.

Univariate and multivariate Cox proportional hazards modeling was used to generate hazard ratio (HR) and corresponding CIs to analyze the association between receipt of surgery and risk of death. The same analysis was also used to identify which sociodemographic factors and clinical variables were associated with OS among the cohort of patients who elected to forego surgery. Variable selection for our multivariate model was completed as described above, and propensity score analysis was completed to minimize confounding. A surgery cohort and a refusal cohort were created using propensity score matching, such that there would be no differences between the two groups in the explanatory covariates. The propensity score matching analysis included a paired matching with a 0.0001 caliper and the following variables: age, race, insurance, geographic region, distant from treatment facility, year of diagnosis, clinical $\mathrm{T}$ category, tumor size, radiation dose, and treatment groups. Additionally, a sensitivity analysis was conducted limited to those patients $<70$ years old with a CCI of 0 to compare survival between the surgery and refusal cohorts. Survival curves were calculated using the Kaplan-Meier method and log-rank test. All statistical analyses were performed using the Stata Statistical Software version 14 (StataCorp. LLC, College Station, TX, USA).

\section{Ethical statement}

The study was conducted in accordance with the Declaration of Helsinki (as revised in 2013). Individual consent for this retrospective analysis was not possible.

\section{Results}

A total of 55,704 patients were eligible for analysis based on our selection criteria: $54,266(97.4 \%)$ received definitive surgery and $1,438(2.6 \%)$ refused surgery. The median age of the entire cohort of patients was 62 years old with a median follow-up time of 123.7 months. Of patients refusing surgery, 135 (9.4\%) were stage I, 709 (49.3\%) were stage II, and 594 (41.3\%) were stage III. Additionally, patients were more likely to decline surgery as the study period progressed on multivariate analysis (OR 1.07, 95\% CI: $1.04-1.10, \mathrm{P}<0.01)$. In $2004,2.1 \%$ of the study population refused surgery, whereas in 2014 that number had increased to $3.3 \%$. Of the 1,438 patients that declined surgery, $238(16.6 \%)$ also declined chemotherapy and 212 (14.7\%) also declined radiotherapy.

There were statistically significant differences on chisquare analysis between the two groups for a majority of the variables analyzed (Table 1). For the entire cohort, a majority of patients were male $(61.9 \%)$, White $(82.2 \%)$, with a CCI of $0(78.4 \%)$, clinical T3 disease $(74.1 \%)$, and received chemotherapy and radiotherapy (80.9\%). In general, patients that received definitive surgery were more likely to be younger, White, have private insurance, and received both chemotherapy and radiotherapy, whereas patients that elected to forego surgery were more likely to be older, have Medicare, and received no chemotherapy or radiotherapy. There were no differences between the two groups based on sex, CCI, percent of residents without a high school degree, facility type, urban density, and clinical $\mathrm{N}$ stage.

Factors predictive of refusing surgery on multivariate analysis included: age 70 years and older compared to the $18-49$ age group $(\mathrm{P}<0.01)$; Black race compared to White race $(\mathrm{P}<0.01)$; uninsured, Medicaid, and Medicare insurance compared to private insurance $(\mathrm{P}<0.01)$; rural facility compared to a metro or urban facility $(\mathrm{P}<0.01)$; tumor size 2.1 to $5.0 \mathrm{~cm}, 5.1$ to $10.0 \mathrm{~cm}$, and greater than $10.0 \mathrm{~cm}$ compared to $2.0 \mathrm{~cm}$ or less $(\mathrm{P}<0.01)$; radiotherapy dose 5,041-5,400 cGy and >5,400 cGy compared to a dose of 5,040 cGy or less $(\mathrm{P} \leq 0.01)$; and radiotherapy treatment alone compared to no chemotherapy or radiotherapy $(\mathrm{P}<0.01)$. Factors predictive of receiving definitive surgery included: treatment in the South compared to the Northeast $(\mathrm{P}<0.01)$; distance of 50 to 200 miles from the treatment facility compared to less than 50 miles from the treatment facility $(\mathrm{P}<0.01)$; clinical $\mathrm{T} 2$ disease compared to clinical $\mathrm{T} 1$ disease $(\mathrm{P}<0.01)$; and treatment with chemotherapy alone or chemotherapy and radiotherapy compared to no treatment $(\mathrm{P}<0.01)$ (Table 2).

Compared to patients that received definitive surgery, 
Table 1 Baseline characteristics by study group

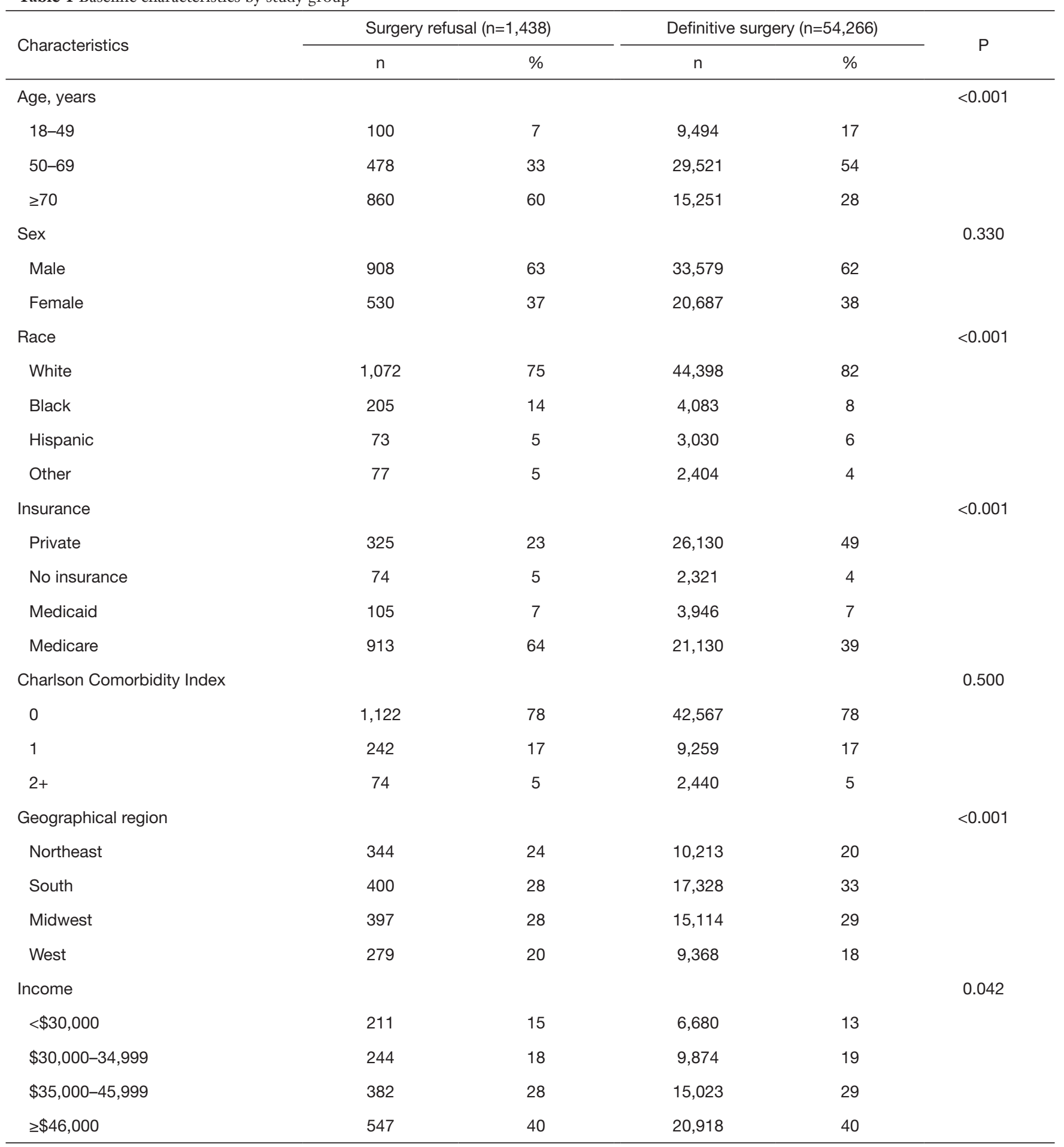

Table 1 (continued) 
Table 1 (continued)

\begin{tabular}{|c|c|c|c|c|c|}
\hline Characteristics & \multicolumn{2}{|c|}{ Surgery refusal $(n=1,438)$} & \multicolumn{2}{|c|}{ Definitive surgery $(n=54,266)$} & $\mathrm{P}$ \\
\hline Residents without high school degree & & & & & 0.339 \\
\hline$\geq 29 \%$ & 246 & 18 & 8,583 & 16 & \\
\hline $20-28.9 \%$ & 309 & 22 & 12,573 & 24 & \\
\hline$<14 \%$ & 491 & 35 & 18,319 & 35 & \\
\hline Facility type & & & & & 0.088 \\
\hline Community & 805 & 57 & 28,303 & 54 & \\
\hline Academic/research & 615 & 43 & 23,720 & 46 & \\
\hline 50 to 200 & 82 & 6 & 5,790 & 11 & \\
\hline$>200$ & 17 & 1 & 946 & 2 & \\
\hline Urban density & & & & & 0.102 \\
\hline Metro/urban & 1,303 & 94 & 49,107 & 93 & \\
\hline Rural & 81 & 6 & 3,687 & 7 & \\
\hline Year of diagnosis & & & & & $<0.001$ \\
\hline 2004 & 67 & 5 & 3,118 & 6 & \\
\hline 2005 & 90 & 6 & 3,391 & 6 & \\
\hline 2011 & 142 & 10 & 5,717 & 11 & \\
\hline 2012 & 143 & 10 & 5,842 & 11 & \\
\hline 2013 & 204 & 14 & 6,164 & 11 & \\
\hline 2014 & 225 & 16 & 6,536 & 12 & \\
\hline Clinical T stage & & & & & $<0.001$ \\
\hline cT1 & 29 & 2 & 662 & 1 & \\
\hline cT2 & 162 & 12 & 9,892 & 19 & \\
\hline cT3 & 1,077 & 77 & 39,006 & 74 & \\
\hline cT4 & 135 & 10 & 3,160 & 6 & \\
\hline
\end{tabular}

Table 1 (continued) 
Table 1 (continued)

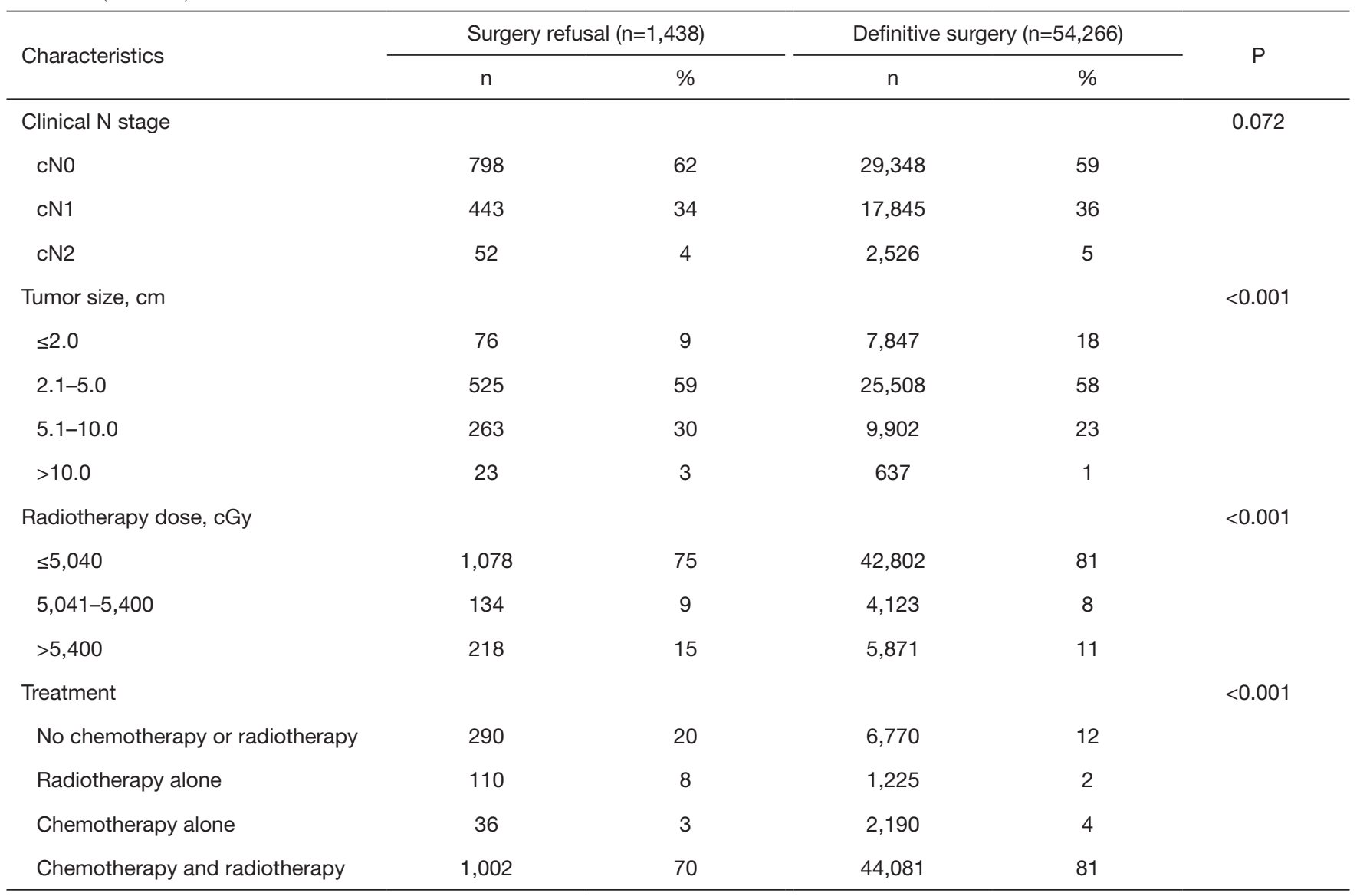

$\mathrm{n}$, number of patients; T, tumor; c, clinical; N, nodes; $\mathrm{cm}$, centimeter; cGy, centigray.

Table 2 Predictors of refusing surgery versus receiving definitive surgery

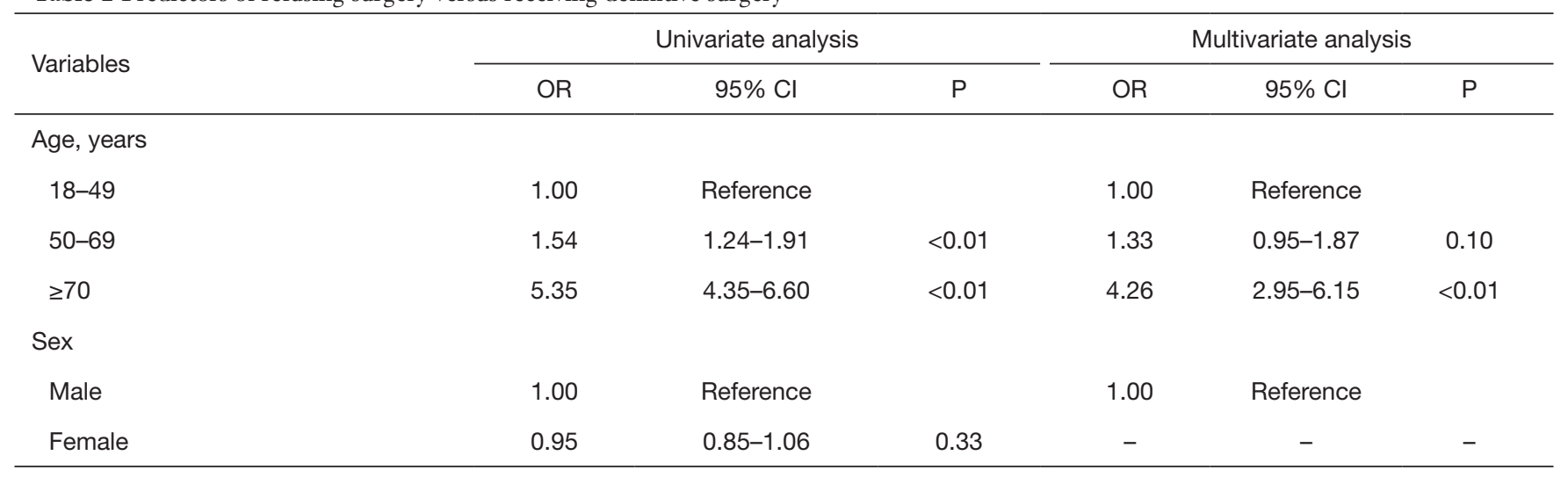

Table 2 (continued) 
Table 2 (continued)

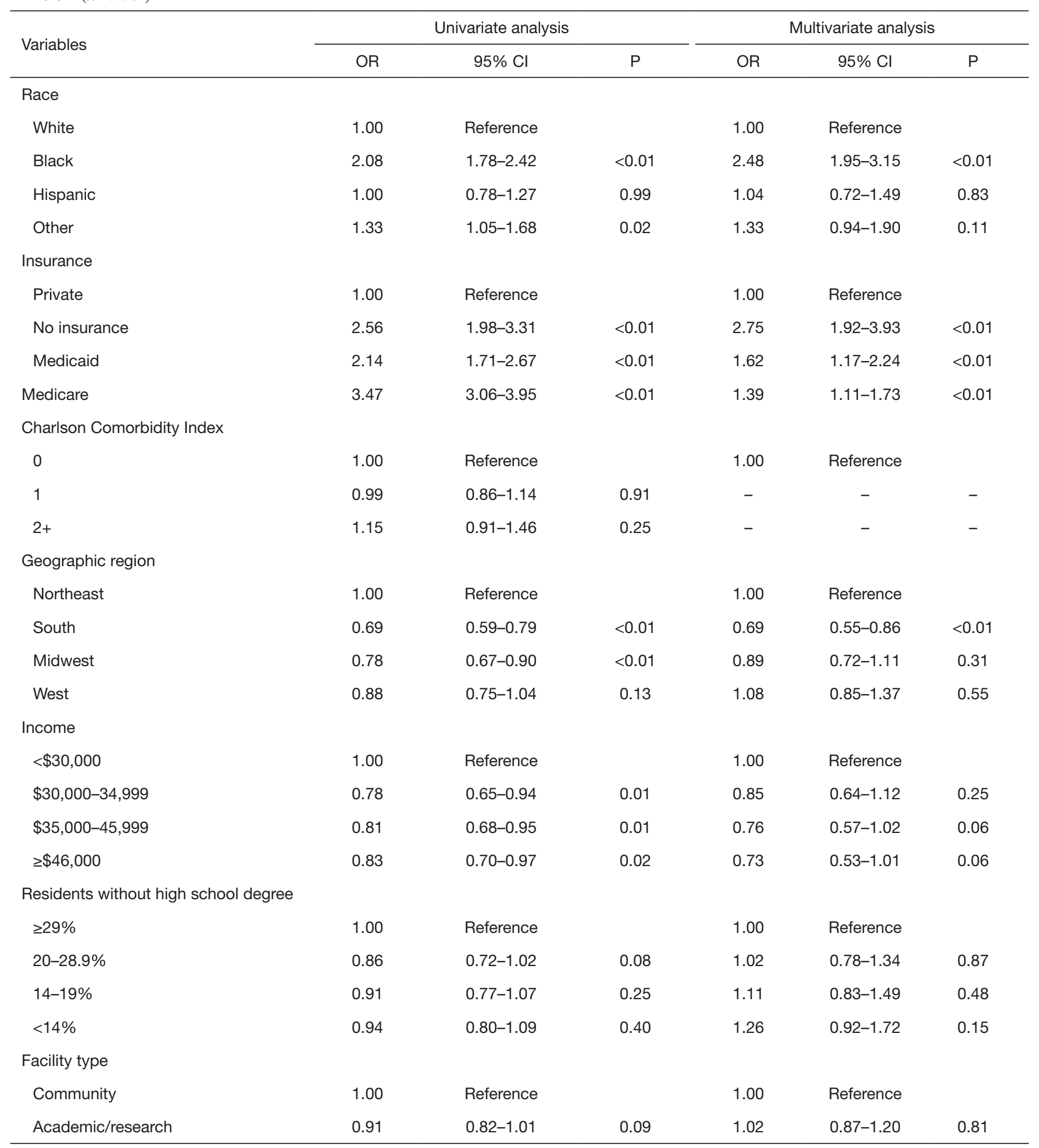

Table 2 (continued) 
Table 2 (continued)

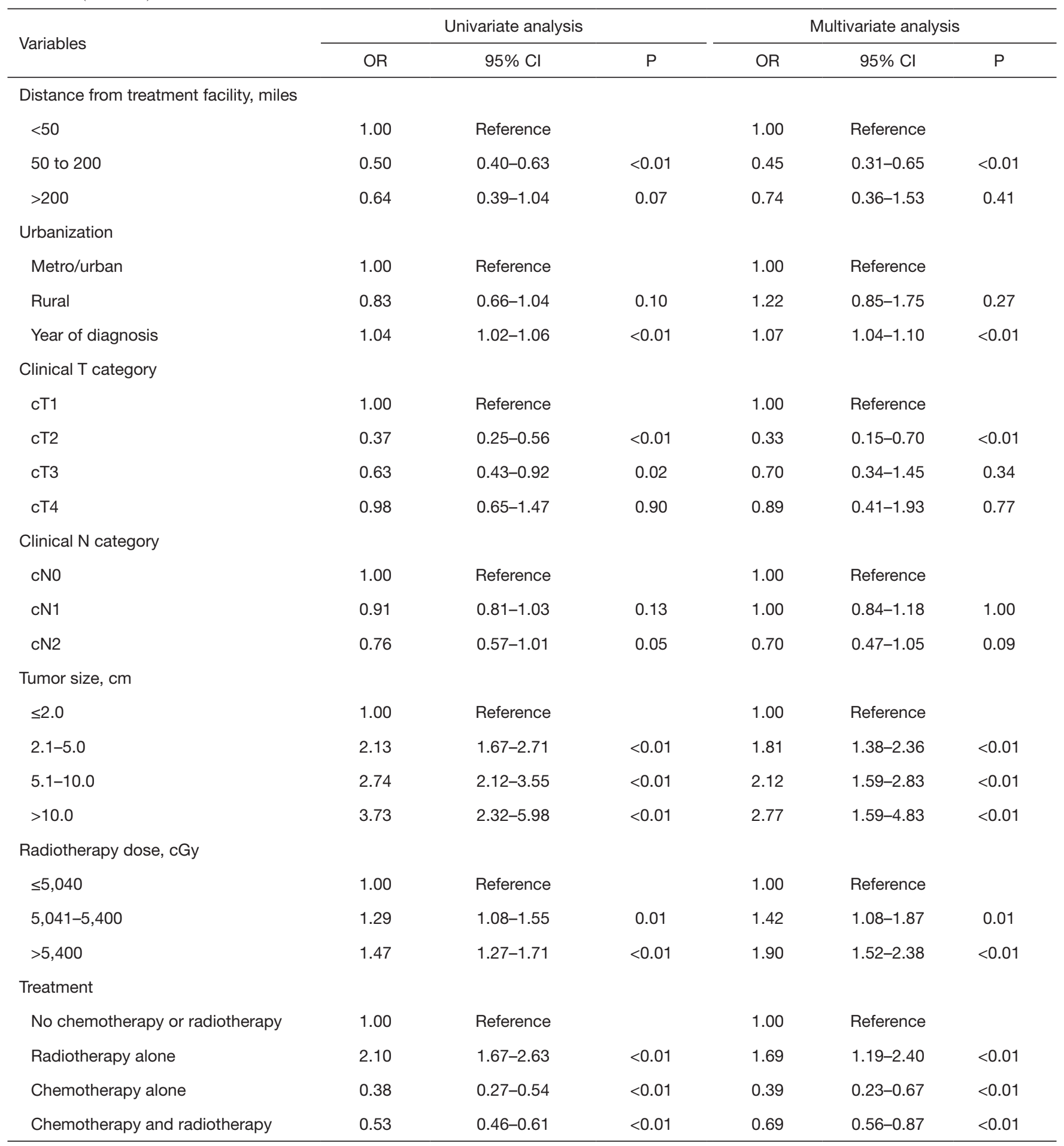

Variables included in the multivariate analysis include: age, race, insurance, geographic region, income, residents without high school degree, facility type, distance from treatment facility, urbanization, year of diagnosis, clinical T category, clinical $\mathrm{N}$ category, tumor size, radiotherapy dose, and treatment groups. OR, odds ratio; $\mathrm{Cl}$, confidence interval; T, tumor; c, clinical; N, nodes; cm, centimeter; cGy, centigray. 


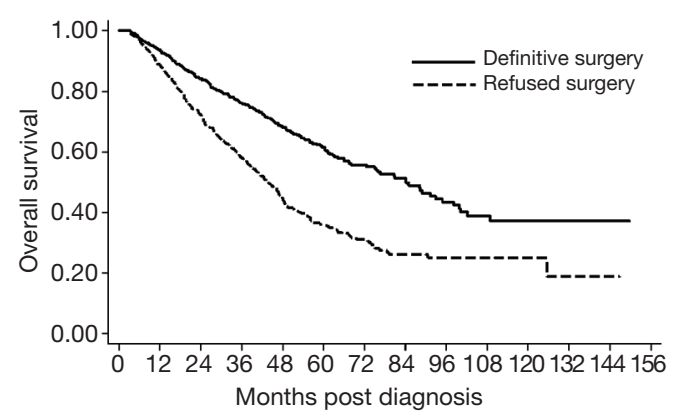

Figure 2 Survival curve comparing propensity-matched patients who received definitive surgery versus patients who refused surgery.

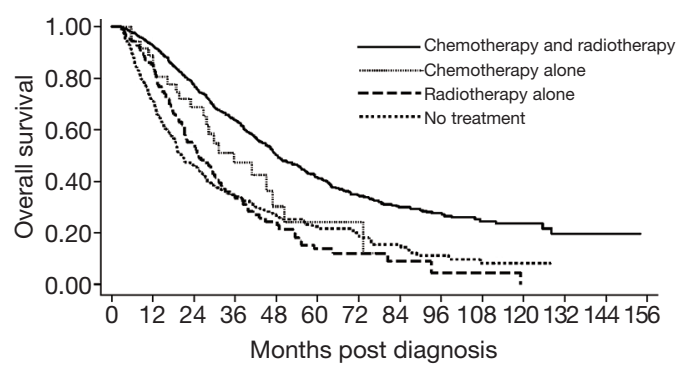

Figure 3 Survival curve for patients that refused surgery, but received chemotherapy and radiotherapy, chemotherapy alone, radiotherapy alone, or no treatment.

patients that declined surgery experienced a significant survival detriment on multivariate analysis (HR 2.32, 95\% CI: 2.09-2.57, $\mathrm{P}<0.01$ ) (Figure S1). These results were consistent analyzing 1,130 patients on propensity score matching showing considerably different survivals for patients who received versus refused surgery (HR 1.91, 95\% CI: 1.60-2.29, $\mathrm{P}<0.001$ ) (Figure 2). The baseline characteristics for the propensity matched groups are shown in the Table S1. The 5 -year OS was $61.6 \%$ for the surgery cohort and $35.7 \%$ for the refusal cohort. Using only the cohorts from the propensity matched analysis, median survival was 84.2 months in patients who received definitive surgery compared to 43.7 months in patients who declined surgery. Among the cohort of patients that elected to forego surgery, those that received chemotherapy and radiotherapy, chemotherapy only, radiotherapy only, or no treatment experienced a median survival of 48.5, 41.0, 28.9, and 18.6 months, respectively ( $\mathrm{P} \leq 0.01$ for all comparisons) (Figure 3). The sensitivity analysis limited to patients who were younger $(<70$ years old) with a CCI of 0 showed a continued survival detriment associated with declining surgery (HR 2.12, 95\% CI: 1.70-2.64, $\mathrm{P}<0.01$ ). In comparison, an analysis limited to patients who were 70 years old and above also showed a survival detriment associated with declining surgery (HR 2.43, 95\% CI: 2.152.75, $\mathrm{P}<0.01)$.

Among only patients that refused surgery, there were variables associated with a survival detriment and benefit. Variables that were associated with a survival detriment on multivariate analysis included: age 70 years and older $(\mathrm{P}<0.01)$, CCI $2+(\mathrm{P}<0.01)$, rural treatment facility $(\mathrm{P}=0.03)$, tumor size 5.0 to $10.0 \mathrm{~cm}(\mathrm{P}=0.02)$, and tumor size $>10.0$ $\mathrm{cm}(\mathrm{P}<0.01)$. Variables that were associated with a survival benefit on multivariate analysis included: other race $(\mathrm{P}<0.01)$ compared to White and treatment with radiotherapy alone $(\mathrm{P}=0.01)$, chemotherapy alone $(\mathrm{P}=0.01)$, or chemotherapy and radiotherapy $(\mathrm{P}<0.01)$ compared to no treatment (Table 3).

\section{Discussion}

In this study, we report the outcomes of patients with nonmetastatic adenocarcinoma of the rectum who received definitive surgery or who refused surgery using a large, hospital-based data set. At NCDB-participating institutions, survival rates for propensity-matched patients who were coded as refusing surgery were much lower compared to patients who received definitive surgery as part of their rectal cancer treatment. This remained true on sensitivity analysis excluding older patients with documented comorbidities. We also discovered sociodemographic and clinical variables associated with declining surgery including: age $\geq 70$ years old, Black race, non-private insurance, rural facility, increasing tumor size, higher radiation dose, and treatment with radiotherapy alone. Patients were also more likely to refuse surgery as the study period progressed.

The decision to forego surgery was a rare event which occurred in only $2.6 \%$ of our study population. The main strength of this study is that we were able to analyze outcomes for a large number of patients for this relatively uncommon clinical situation. It is important to note that the decision-making process regarding treatment between the patient and their health care provider is complex with nuance that is difficult to capture in an aggregate database. There are many potential reasons or clinical scenarios that may lead a patient to decline surgery. Our goal is to provide outcomes data to help guide the conversation for these difficult decisions. Our results are consistent with other studies, which illustrated a survival detriment for patients that refused surgery for other disease sites (11-14). Among 
Table 3 Predictors of overall survival in patients who refused surgery

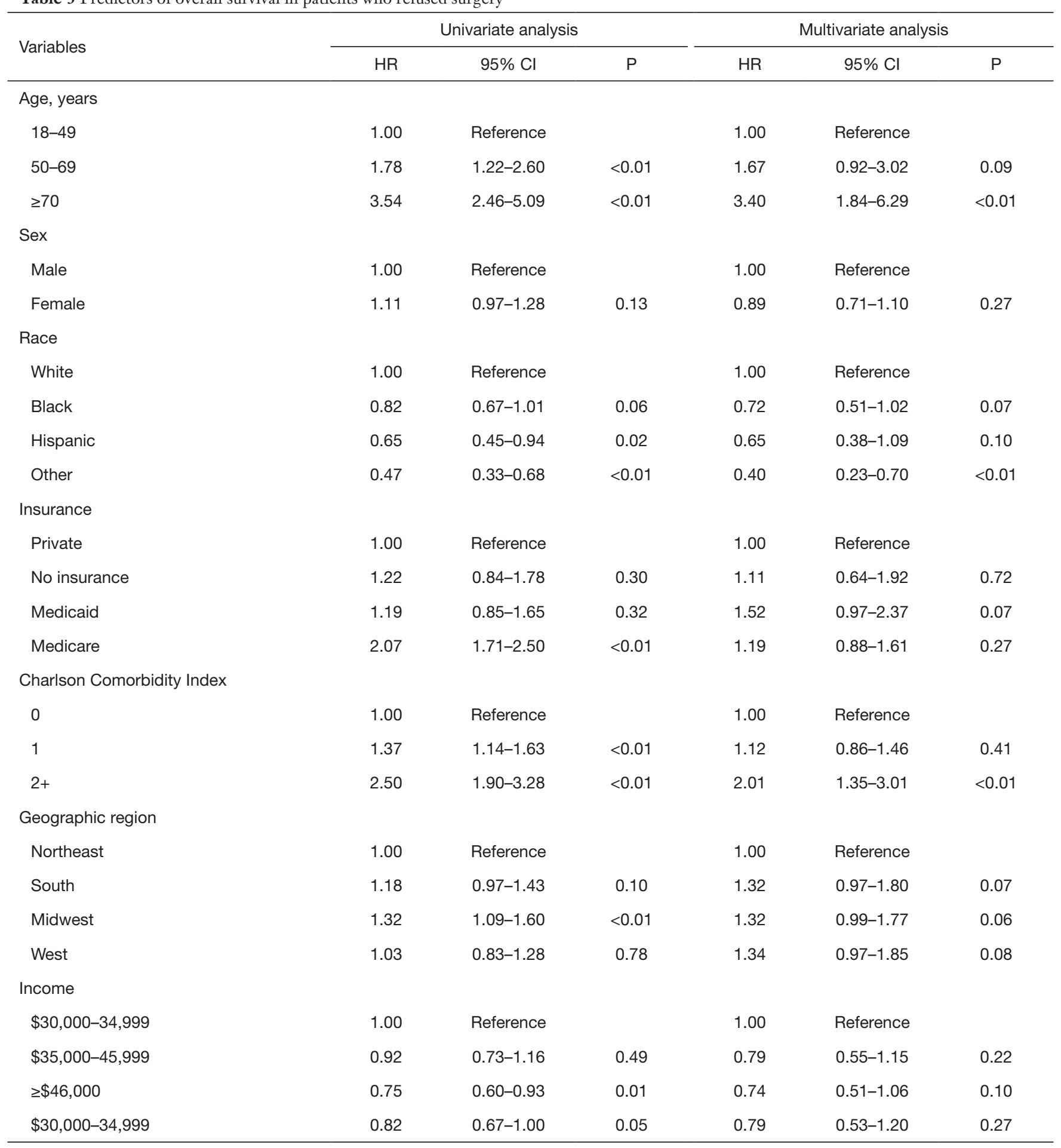

Table 3 (continued) 
Table 3 (continued)

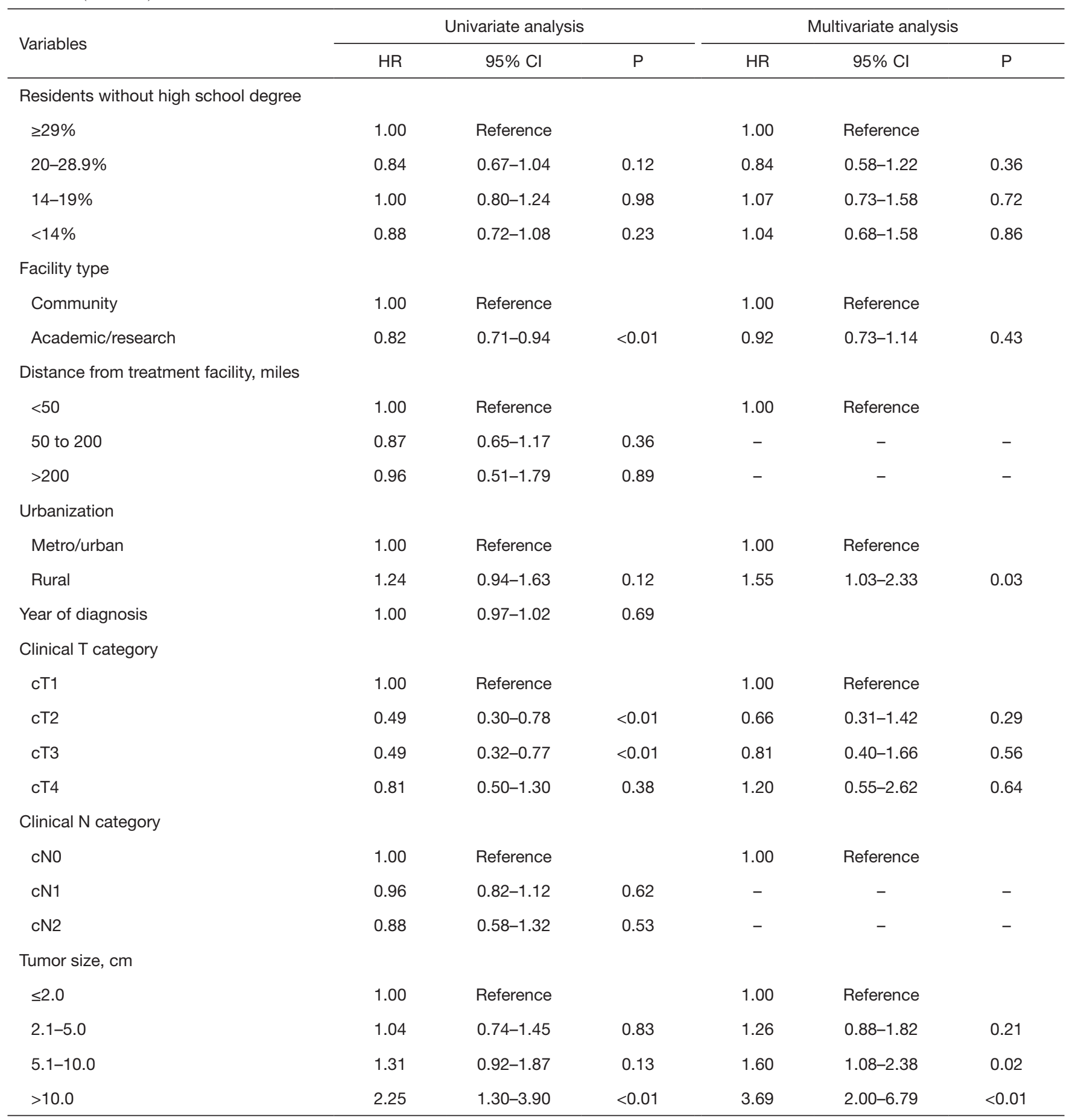

Table 3 (continued) 
Table 3 (continued)

\begin{tabular}{|c|c|c|c|c|c|c|}
\hline Variables & \multicolumn{3}{|c|}{ Univariate analysis } & \multicolumn{3}{|c|}{ Multivariate analysis } \\
\hline \multicolumn{7}{|l|}{ Radiotherapy dose, cGy } \\
\hline$\leq 5,040$ & 1.00 & Reference & & 1.00 & Reference & \\
\hline $5,041-5,400$ & 0.94 & $0.74-1.19$ & 0.61 & - & - & - \\
\hline \multicolumn{7}{|l|}{ Treatment } \\
\hline No chemotherapy or radiotherapy & 1.00 & Reference & & 1.00 & Reference & \\
\hline Radiotherapy alone & 0.99 & $0.77-1.27$ & 0.92 & 0.63 & $0.43-0.91$ & 0.01 \\
\hline Chemotherapy alone & 0.70 & $0.45-1.09$ & 0.12 & 0.35 & $0.17-0.74$ & 0.01 \\
\hline
\end{tabular}

Variables included in the multivariate analysis include: age, sex, race, insurance, Charlson comorbidity index, geographic region, income, residents without high school degree, facility type, urbanization, clinical T category, tumor size, and treatment groups. HR, hazard ratio; Cl, confidence interval; T, tumor; c, clinical; N, nodes; cm, centimeter; cGy, centigray.

these studies that show decreased survival for patients that decline surgery, only one provides some insight into the reasoning. In breast cancer, the most common reasons for refusing surgery include psychiatric problems (mostly depression), pursuit of alternative therapy, and other medical problems (12). One study analyzed patients that declined conventional cancer treatment in general, which included reasons such as fear of side effects, emotional impact of diagnosis, poor communication, and control over the decision-making process (15). However, research is severely limited in exploring the reasoning and motivation behind a patient's decision to forego cancer surgery or cancer treatment in general.

The median survival for patients that received definitive surgery on propensity matched analysis was approximately twice as long as for those who declined surgery. This validates the importance of surgery with regard to survival in the treatment paradigm for non-metastatic rectal cancer. Among the cohort of patients that elected to forego surgery, those that received chemotherapy and radiotherapy, chemotherapy only, or radiotherapy only experienced a statistically significant improvement in survival compared to patients that received no treatment, but the improvements are modest compared to the surgery cohort. The survival outcomes were consistent using propensity score matching in an attempt to minimize confounding variables. These data do not imply that surgical resection is the only treatment approach that can provide long-term survival in rectal cancer. The "watch and wait" organ preservation strategy may eventually reveal a subset of patients that can be cured without surgery, but the current treatment paradigm that leads to long-term survival is based on surgery. Also, a planned watch and wait approach would be expected to result in vastly different outcomes than refusing a recommended surgery.

Many variables were associated with declining surgery including advanced age, Black race, non-private insurance, and increasing tumor size. Interestingly, these factors were also found to be associated with refusing oncologic surgery in patients with pancreatic cancer (13). The association between older age and refusing cancer treatments has been shown in other studies (20-22). Interestingly, while older patients were more likely to refuse surgery, those who did still experienced a survival detriment as shown in our subset analysis. Black patients have also been shown to refuse surgery at higher rates in other disease sites $(23,24)$. The finding that non-private insurance is associated with refusal of surgery raises the important question of whether the ability to pay is directing patients away from important cancer treatments.

Increasing radiation dose was associated with declining surgery and it is possible that a higher dose was being used in order to compensate for a lack of surgery. Even though receipt of radiotherapy alone or a combination of chemotherapy and radiotherapy was associated with an improvement in survival, the numbers are small compared to 
the definitive surgery cohort. This information is important for patients that are not surgical candidates, but if surgery is an option, it suggests that surgery should be strongly considered for long-term survival. Another component of the surgery is the type of surgery performed. Patients with low-lying tumors often require an abdominoperineal resection (APR) for definitive treatment. We postulate that patients who require an APR would be more likely to refuse surgery as a permanent colostomy is required, even though multiple studies in contemporary cohorts have shown similar or worse quality of life after sphincter preservation (25-27). This specific group of patients was not able to be studied using the data that is available in the NCDB.

A potentially troubling discovery is the statistically significant trend of increasing refusal of surgery as the study period progressed. This trend has also been shown recently in breast cancer surgery (22). Our study offers no explanation as to why more patients are declining surgery, but it is certainly a trend that should be monitored closely in an attempt to better understand the reasons why. A potential reason as to why patients seem to be refusing surgery more frequently may be related to knowledge of the "watch and wait" approach. It is possible that some patients prefer organ preservation, but might not necessarily be the best candidates. These patients may elect for "watch and wait" even though their treatment team is recommending surgical intervention. Certainly, the survival data presented here should be strongly considered in the treatment decisionmaking process and incorporated into the numerous other variables that factor into the treatment decision.

There are many variables associated with decreased survival among the refusal cohort. Advanced age, CCI 2+, and increasing tumor size are all associated with worse survival, consistent with rectal cancer patients in general. The reasons for rural location being associated with a survival detriment are less clear, but could be explained by decreased proximity to supportive care or other resources like chemotherapy and radiation therapy. Patients at rural facilities were also more likely to decline surgery, perhaps due to unwillingness to travel for treatments.

The limitations of this study are similar to other population-based retrospective reviews. Propensity match analysis can help minimize the selection bias with known variables, but does not account for unknown variables, such as surgical fitness, patient values, or other factors considered during nuanced rectal cancer treatment. Also, this study population only reflects the institutions who participate in NCDB.
In conclusion, we present the expected survival outcomes for patients who refused surgery for non-metastatic adenocarcinoma of the rectum using a large populationbased dataset. There is a significant survival detriment in patients who elected to forego surgical resection, which can be improved modestly with chemotherapy and/ or radiotherapy. This information could be used in the decision-making process when the provider and patient are weighing the benefits and potential side effects of treatment. The trend of declining cancer surgery should be further investigated.

\section{Acknowledgments}

Funding: None.

\section{Footnote}

Reporting Checklist: The authors have completed the STROBE reporting checklist (available at https://dx.doi. org/10.21037/jgo-20-437).

Peer Review File: Available at https://dx.doi.org/10.21037/ jgo-20-437

Conflicts of Interest: All authors have completed the ICMJE uniform disclosure form (available at https://dx.doi. org/10.21037/jgo-20-437). RT reports personal fees from QED Therapeutics and personal fees from the Lynx Group, which are both outside of the submitted work. The other authors have no conflicts of interest to declare.

Ethical Statement: The authors are accountable for all aspects of the work in ensuring that questions related to the accuracy or integrity of any part of the work are appropriately investigated and resolved. The study was conducted in accordance with the Declaration of Helsinki (as revised in 2013). This study was exempt from oversight by the Institutional Review Board as there is no identifiable patient information in the NCDB. Individual consent for this retrospective study was not possible.

Open Access Statement: This is an Open Access article distributed in accordance with the Creative Commons Attribution-NonCommercial-NoDerivs 4.0 International License (CC BY-NC-ND 4.0), which permits the noncommercial replication and distribution of the article with the strict proviso that no changes or edits are made and the 
original work is properly cited (including links to both the formal publication through the relevant DOI and the license). See: https://creativecommons.org/licenses/by-nc-nd/4.0/.

\section{References}

1. Siegel RL, Miller KD, Fuchs HE, et al. Cancer Statistics, 2021. CA Cancer J Clin 2021;71:7-33.

2. Habr-Gama A, Perez RO, Nadalin W, et al. Operative versus nonoperative treatment for stage 0 distal rectal cancer following chemoradiation therapy: long-term results. Ann Surg 2004;240:711-7; discussion 717-8.

3. Habr-Gama A, Sabbaga J, Gama-Rodrigues J, et al. Watch and wait approach following extended neoadjuvant chemoradiation for distal rectal cancer: are we getting closer to anal cancer management? Dis Colon Rectum 2013;56:1109-17.

4. Maas M, Beets-Tan RG, Lambregts DM, et al. Waitand-see policy for clinical complete responders after chemoradiation for rectal cancer. J Clin Oncol 2011;29:4633-40.

5. Appelt AL, Pløen J, Harling H, et al. High-dose chemoradiotherapy and watchful waiting for distal rectal cancer: a prospective observational study. The Lancet Oncology 2015;16:919-27.

6. Renehan AG, Malcomson L, Emsley R, et al. Watchand-wait approach versus surgical resection after chemoradiotherapy for patients with rectal cancer (the OnCoRe project): a propensity-score matched cohort analysis. The Lancet Oncology 2016;17:174-83.

7. National Comprehensive Cancer Network. Rectal Cancer, Version 1. 2019. Available online: https://www.nccn.org/ professionals/physician_gls/pdf/rectal.pdf

8. Steele GD, Jr., Herndon JE, Bleday R, et al. Sphinctersparing treatment for distal rectal adenocarcinoma. Ann Surg Oncol 1999;6:433-41.

9. Greenberg JA, Shibata D, Herndon JE, 2nd, et al. Local excision of distal rectal cancer: an update of cancer and leukemia group B 8984. Dis Colon Rectum 2008;51:118591; discussion 91-4.

10. Russell AH, Harris J, Rosenberg PJ, et al. Anal sphincter conservation for patients with adenocarcinoma of the distal rectum: long-term results of radiation therapy oncology group protocol 89-02. Int J Radiat Oncol Biol Phys 2000;46:313-22.

11. Wang J, Wang FW. Refusal of cancer-directed surgery strongly impairs survival of patients with localized hepatocellular carcinoma. Int J Surg Oncol
2010;2010:381795.

12. Verkooijen HM, Fioretta GM, Rapiti E, et al. Patients' refusal of surgery strongly impairs breast cancer survival. Ann Surg 2005;242:276-80.

13. Coffman A, Torgeson A, Lloyd S. Correlates of Refusal of Surgery in the Treatment of Non-metastatic Pancreatic Adenocarcinoma. Ann Surg Oncol 2019;26:98-108.

14. Massa ST, Osazuwa-Peters N, Franco J, et al. Survival after refusal of surgical treatment for locally advanced laryngeal cancer. Oral Oncol 2017;71:34-40.

15. Verhoef MJ, Rose MS, White M, et al. Declining conventional cancer treatment and using complementary and alternative medicine: a problem or a challenge? Curr Oncol 2008;15 Suppl 2:s101-6.

16. Hupkens BJP, Martens MH, Stoot JH, et al. Quality of Life in Rectal Cancer Patients After Chemoradiation: Watch-and-Wait Policy Versus Standard Resection - A Matched-Controlled Study. Dis Colon Rectum 2017;60:1032-40.

17. Surgeons ACo. National Cancer Database. Available online: https://www.facs.org/quality-programs/cancer/ ncdb.

18. Maldonado G, Greenland S. Simulation study of confounder-selection strategies. Am J Epidemiol 1993;138:923-36.

19. Budtz-Jørgensen E, Keiding N, Grandjean P, et al. Confounder selection in environmental epidemiology: assessment of health effects of prenatal mercury exposure. Ann Epidemiol 2007;17:27-35.

20. Liu CY, Chen WT, Kung PT, et al. Characteristics, survival, and related factors of newly diagnosed colorectal cancer patients refusing cancer treatments under a universal health insurance program. BMC Cancer 2014;14:446.

21. Aizer AA, Chen MH, Parekh A, et al. Refusal of curative radiation therapy and surgery among patients with cancer. Int J Radiat Oncol Biol Phys 2014;89:756-64.

22. Gaitanidis A, Alevizakos M, Tsalikidis C, et al. Refusal of Cancer-Directed Surgery by Breast Cancer Patients: Risk Factors and Survival Outcomes. Clin Breast Cancer 2018;18:e469-76.

23. Lathan CS, Neville BA, Earle CC. The effect of race on invasive staging and surgery in non-small-cell lung cancer. J Clin Oncol 2006;24:413-8.

24. Cykert S, Dilworth-Anderson P, Monroe MH, et al. Factors associated with decisions to undergo surgery among patients with newly diagnosed early-stage lung cancer. JAMA 2010;303:2368-76. 
25. Feddern ML, Emmertsen KJ, Laurberg S. Quality of life with or without sphincter preservation for rectal cancer. Colorectal Dis 2019;21:1051-7.

26. How P, Stelzner S, Branagan G, et al. Comparative quality of life in patients following abdominoperineal excision and

Cite this article as: Coffman AR, Tao R, Cohan JN, Huang LC, Pickron TB, Torgeson AM, Lloyd S. Factors associated with the refusal of surgery and the associated impact on survival in patients with rectal cancer using the National Cancer Database. J Gastrointest Oncol 2021;12(4):1482-1497. doi: 10.21037/jgo-20-437 low anterior resection for low rectal cancer. Dis Colon Rectum 2012;55:400-6.

27. Pachler J, Wille-Jørgensen P. Quality of life after rectal resection for cancer, with or without permanent colostomy. Cochrane Database Syst Rev 2004;(3):CD004323. 